\title{
Searches for dark photons in $\mathrm{LHCb}$
}

\author{
Adrián Casais Vidal* \\ on behalf of the LHCb Collaboration \\ Instituto Galego de Física de Altas Enerxías, Universidade de Santiago de Compostela \\ E-mail: adrian.casais.vidalecern.ch
}

LHCb, while designed mainly for physics involving $b$ quarks has lately become a general purpose detector in the forward region. Focusing on searches for dark photons, some of the most relevant $\mathrm{LHCb}$ results and prospects concerning dark sectors will be presented.

7th Annual Conference on Large Hadron Collider Physics - LHCP2019

20-25 May, 2019

Puebla, Mexico

${ }^{*}$ Speaker. 


\section{Introduction}

LHCb is a single arm spectrometer designed to cover the high rapidity region: $2<\eta<5$ [1]. Although it was originally built to study heavy flavor physics, it is currently functioning as a general purpose detector in the forward region. This broad physics scope includes results concerning dark sectors.

Dark sectors are scenarios in which the New Physics (NP) structure appears somehow disentangled from the Standard Model of particle physics (SM). In this scenario, portals generated by Quantum Mechanics could be mechanisms of communication for these sectors and the SM. LHCb has performed searches using the following portals:

- Vector portal $\left(A^{\prime}\right)$

- Scalar portal $(H)$

- Axion portal (a)

- Neutrino portal

In these proceedings, the vector portal results obtained by $\mathrm{LHCb}$, in the form of searches for dark photons, will be discussed. A search for a dimuon resonance in the $\Upsilon$ mass region, which concerns the scalar portal, will also be shown. Finally, prospects for the search for Axion-Like particles (ALPs), related to the axion portal will be briefly discussed.

\section{Dark photons}

LHCb has performed a search for dark photons, $A^{\prime}$, produced in $p p$ collisions at $\sqrt{s}=13 \mathrm{TeV}$ and decaying into two muons: $A^{\prime} \rightarrow \mu^{+} \mu^{-}$[2].

Dark photons are produced via kinetic mixing $\gamma-A^{\prime}$ and inherit the production mechanisms of off-shell photons $\left(\gamma^{*}\right)$. This feature makes dark photon candidates kinematically identical to virtual photons. Depending on the lifetime of the dark photon two searches were performed. Firstly, a prompt-like search in a mass range going from the dimuon threshold up to the $Z$ boson pole $\left(2 m_{\mu}<\right.$ $\left.m\left(A^{\prime}\right)<70 \mathrm{GeV}\right)$. Secondly, a displaced search was performed in a sensitive region spanning from near threshold: $214 \mathrm{MeV}<m\left(A^{\prime}\right)<350 \mathrm{MeV}$. The displaced search is the first result of this kind not coming from a beam dump experiment.

Some of the features of the $\mathrm{LHCb}$ detector that that are key for this search are the following: good muon identification to minimize pion misidentification; good vertexing to remove muons coming from displaced decays, mostly from heavy flavour; and also its good mass resolution for being able to to identify a mass peak on top of the dimuon continuum.

\subsection{Prompt search}

An event selection was performed based on $p_{T}$ requirements and particle identification in the mass range $2 m_{\mu}<m\left(A^{\prime}\right)<70 \mathrm{GeV}$. The background contributions come from different components: true muons produced by off-shell photons (which cannot be reduced), light hadrons misreconstructed as muons and muons from the decay chain of heavy flavor hadrons misidentified as 
prompt. To separate the true prompt dimuons from the two latter sources of background, fits relying on the quality of the vertices were performed, as shown in Fig.1 (Top). Prompt dimuon templates are taken from different prompt resonances and heavy QCD background sources were taken from same sign muons as well as simulation. A correction to the normalization of this was obtained using dipion data samples.

A scan for signal was performed on top of the prompt dimuon component. Since no significant excess was found upper limits on the number of observed events were placed at the different masses. 90\% confidence level (CL) exclusion regions on the $\varepsilon^{2}-m\left(A^{\prime}\right)$ plane obtained which can be seen in Fig.1 (Bottom).
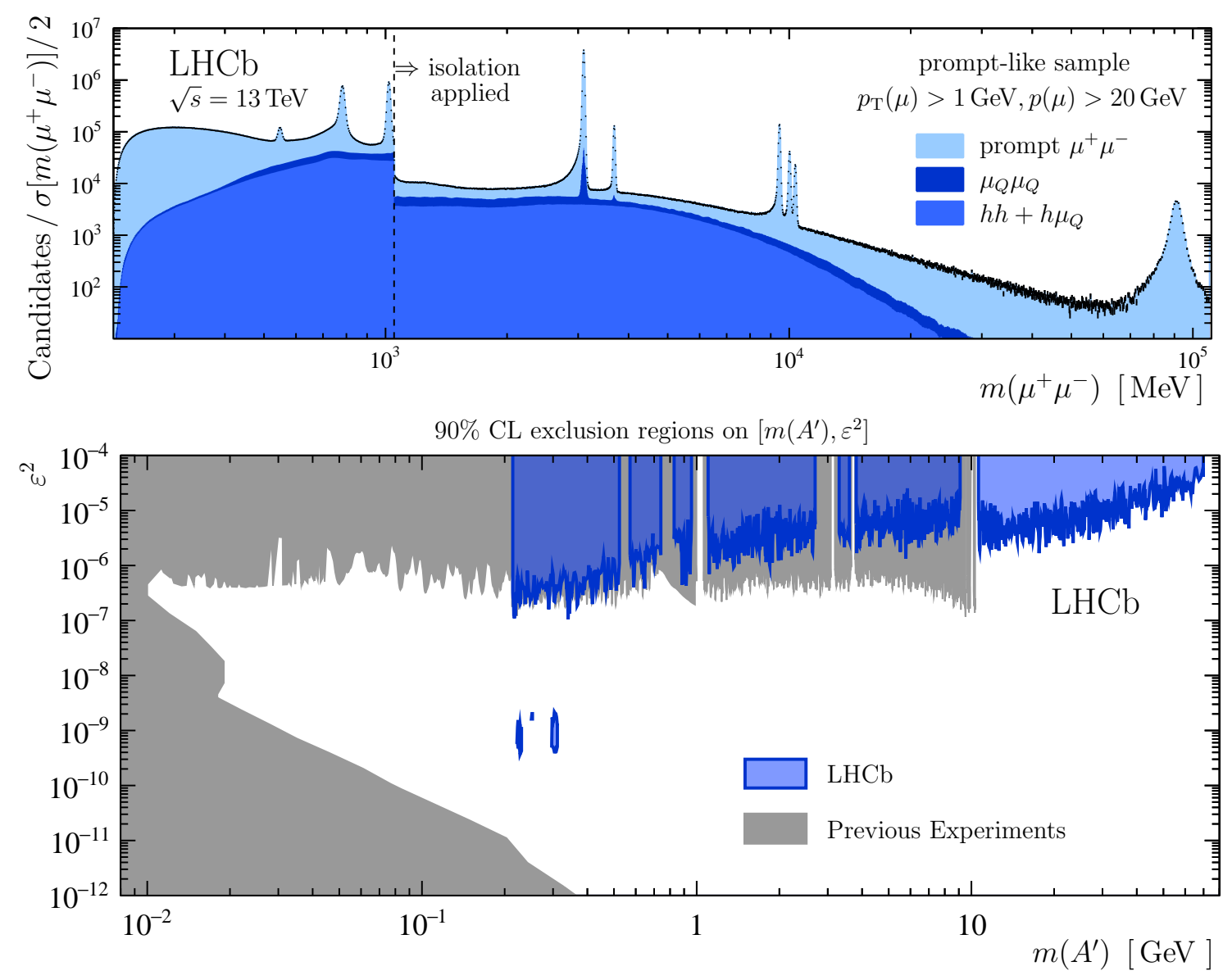

Figure 1: Top: spectrum of dimuon candidates. Bottom: exclusion regions on the dark photons parameter space

\subsection{Displaced search}

This search was performed in a smaller mass region: $214 \mathrm{MeV}<m\left(A^{\prime}\right)<350 \mathrm{MeV}$. In this case the background is smaller and dominated by photon conversions to dimuons in the material of the Vertex Locator (VELO). A method to identify particles produced in secondary interactions in the VELO was developed [3]. Using a high precision three-dimensional map of secondary interactions, produced with this method, as well as characteristics from the $A^{\prime} \rightarrow \mu^{+} \mu^{-}$decay, 
the photon conversion yield is reduced to insignificant levels. The search for a signal excess was performed similarly to the prompt search. Some differences arise from the fact that the lifetime of the candidates must be taken into account. In this case the scan was performed in discrete steps in $m\left(A^{\prime}\right)$ and also $\tau\left(A^{\prime}\right)$. No significant excess was found and exclusion regions obtained in previously unexplored parameter space can be seen in Fig.1 (Bottom). These are the first limits obtained on long lived dark photons not coming from beam dump experiments.

\subsection{Future reach}

LHCb has shown its unique sensitivity to search dark photons into dimuons in a dedicated search done with Run 2 data. Using the knowledge cast by this analysis, the low-mass dark photon efficiency has been improved for 2017 data taking. Looking deeper into the future, by Run 3 data taking, LHCb will have removed its hardware level trigger, which, together with the higher expected luminosity, should increase the number of expected $A^{\prime} \rightarrow \mu^{+} \mu^{-}$by two or even three orders of magnitude.

Furthermore, to explore currently unconstrained dark photon parameter space, charmed meson decays can be used: $D^{0 *} \rightarrow D^{0} e^{+} e^{-}$[4]. This channel opens the possibility to look below the dimuon threshold. Thanks to the triggerless readout in Run 3, $\mathscr{O}\left(10^{10}\right) D^{0 *} \rightarrow D^{0} \gamma^{*}$ per $\mathrm{fb}^{-1}$ are expected. The expected limits for the different upgrade phases can be seen at Fig. 2

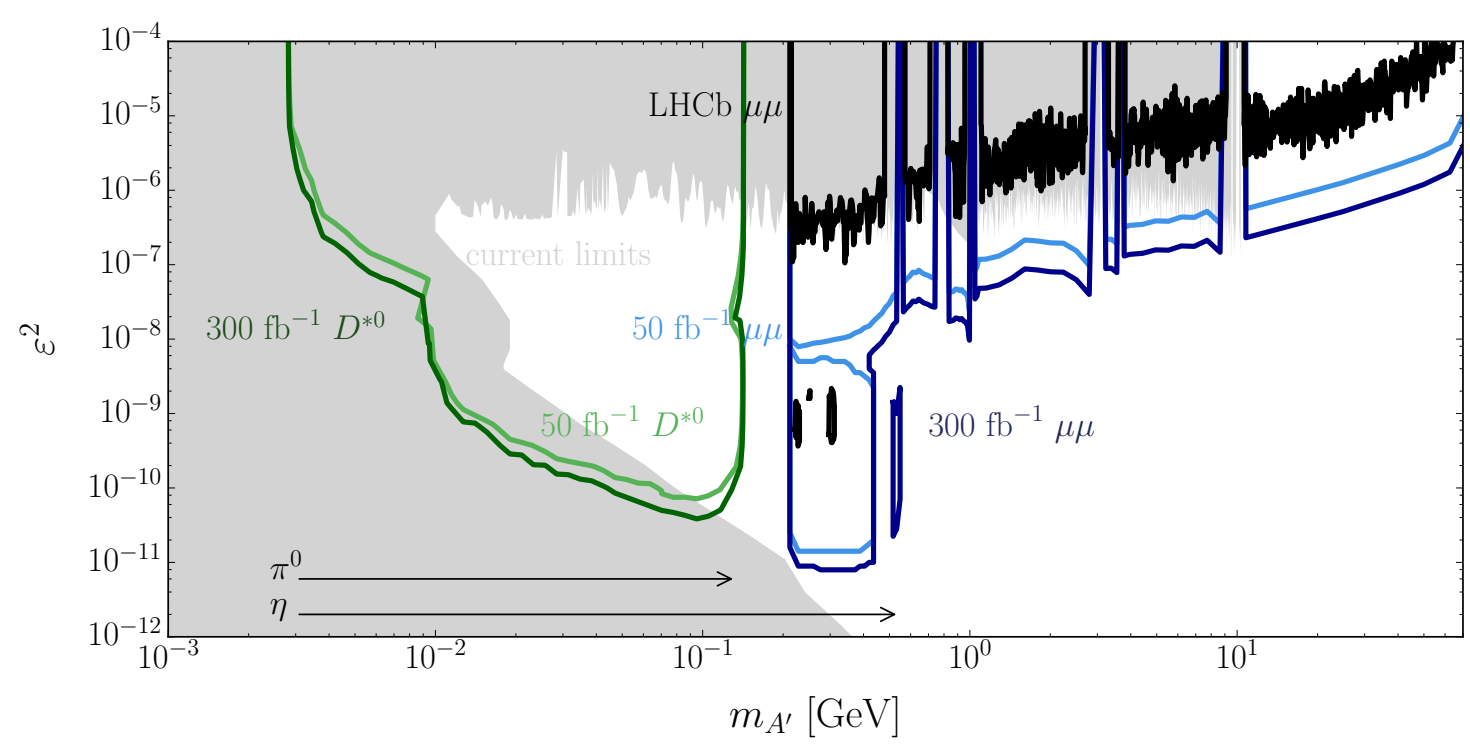

Figure 2: Black: regions currently excluded by LHCb. Blue: future reach for the dimuon strategy. Green: expected limits using the $D^{0 *} \rightarrow D^{0} e^{+} e^{-}$decay in Run 3.Grey: limits placed by other experiments.

\section{Search for a dimuon resonance in the $\Upsilon$ region}

LHCb has performed a model independent search for a dimuon resonance produced in $p p$ collisions in the mass range between 5.5 and $15 \mathrm{GeV}$ using data corresponding to an integrated 

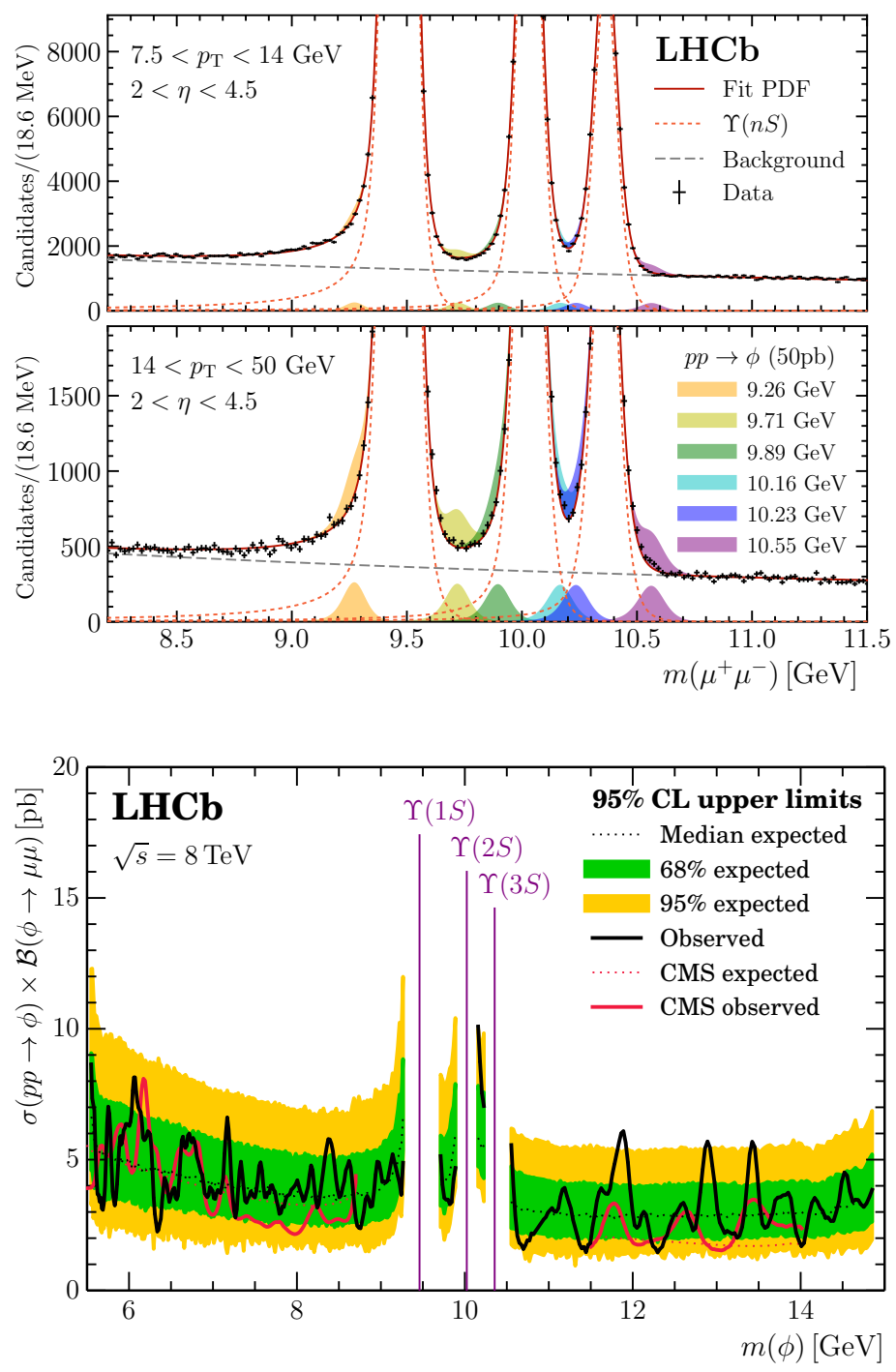

Figure 3: (Top) results on the mass fit over the dimuon spectrum. (Bottom) Limits on the cross section times the branching fraction for a spin 0 boson produced in gluon fusion.

luminosity of 0.98 (1.99) $\mathrm{fb}^{-} 1$ taken during 2011 (2012) at $\sqrt{s}=7$ (8) $\mathrm{TeV}$ [5]. This search benefits from different $\mathrm{LHCb}$ features in the same way as the dark photon search (Section 2).

The search is performed in the context of a spin- 0 boson $\phi$ produced directly in $p p$ collisions via gluon-gluon fusion. The analysis is split in kinematic bins to improve the sensitivity in different reinterpretations of the results.

Searching near the bottonium resonances and even between the three main resonances $\Upsilon(1 S)$, $\Upsilon(2 S), \Upsilon(3 S)$ was possible thanks to the LHCb mass resolution and the precise modelling of the mass tails of the $\Upsilon$ resonances. The results of the fit to the different mass hypotheses as well as the limits in the cross-section times branching fraction can be found in Fig. 3. 


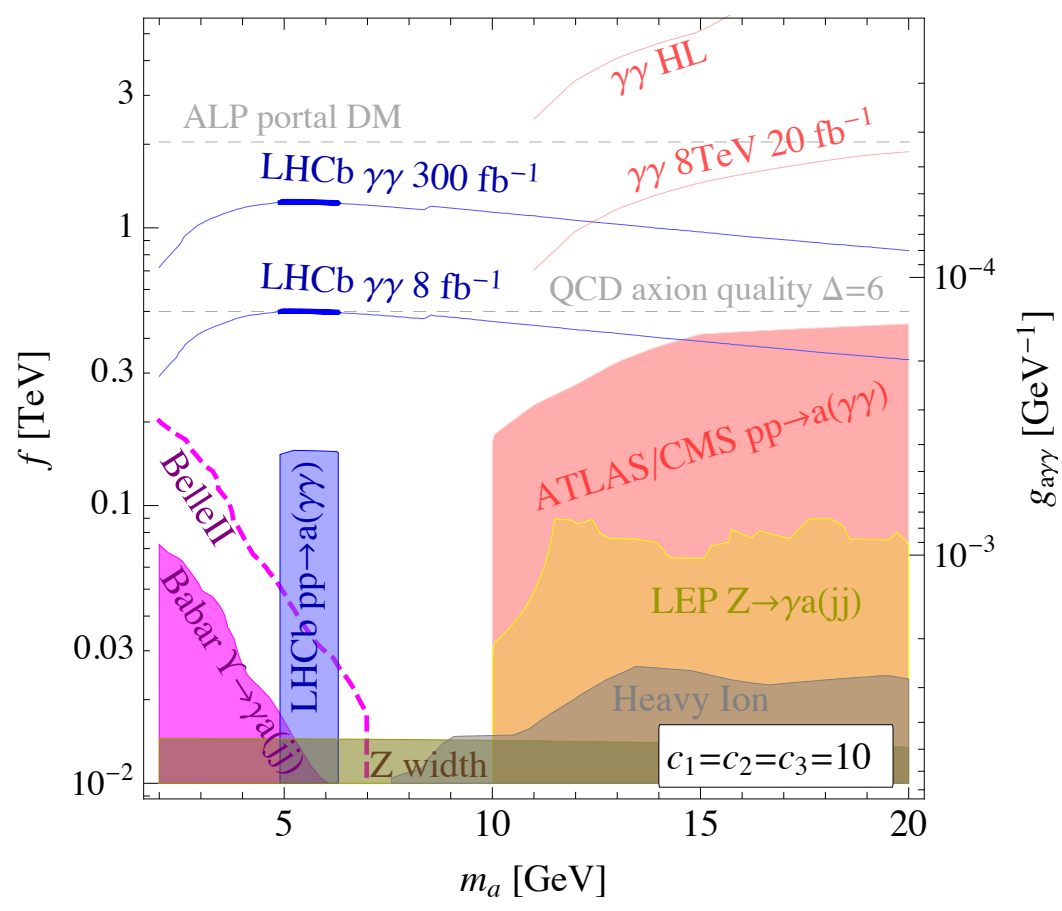

Figure 4: Current exclusion regions on the parameter space of ALPs. LHCb placed the best limits on a portion of the "gap" between 5 and $10 \mathrm{Gev}$.

\section{Future search for axion like particles}

Axion like particles are pseudo Nambu-Goldstone bosons associated to the spontaneous breaking of partial symmetries. An interesting scenario is one in which the energy scale of NP, $M_{N P}$ lies beyond the LHC reach. In that case, ALPs can be lighter than NP scale, $M_{a}<<M_{N P}$, with the decay rate of ALPs being controlled by the inverse of its decay constant, $1 / f$ and the NP scale being proportional to this "coupling": $M_{N P}=f g_{*}$, where $g_{*}$ is the typical size of the coupling of NP sectors to the SM. This relation opens a window to place constraints on the NP scale by looking at the coupling strength of ALPs to the SM [6].

The interest of $\mathrm{LHCb}$ is focused in a mass window of $3-10 \mathrm{GeV}$, which lies inside the allowed dynamic range of the $\mathrm{LHCb}$ electromagnetic calorimeter. A recast of a subset of $\mathrm{LHCb}$ data, corresponding to an integrated luminosity of $80 \mathrm{pb}^{-1}$, has placed the best limits in a portion of this mass window for ALPs decaying into two photons [6] (Fig. 4).

\section{Conclusions}

LHCb has shown its unique sensitivity for searches in fields which diverge from its design case: heavy flavor physics. World-best results in dark sector scenarios have been delivered and unique sensitivity has been shown, leaving room for improvement in the forthcoming upgrades of the detector. 


\section{References}

[1] LHCB collaboration, The LHCb Detector at the LHC, JINST 3 (2008) S08005.

[2] LHCB collaboration, Search for Dark Photons Produced in $13 \mathrm{TeV}$ pp Collisions, Phys. Rev. Lett. 120 (2018) 061801 [1710.02867].

[3] M. Alexander et al., Mapping the material in the LHCb vertex locator using secondary hadronic interactions, JINST 13 (2018) P06008 [1803.07466].

[4] Working Group 3 collaboration, Beyond the Standard Model Physics at the HL-LHC and HE-LHC, 1812.07831.

[5] LHCB collaboration, Search for a dimuon resonance in the ؟ mass region, JHEP 09 (2018) 147 [1805.09820].

[6] X. Cid Vidal, A. Mariotti, D. Redigolo, F. Sala and K. Tobioka, New Axion Searches at Flavor Factories, JHEP 01 (2019) 113 [1810.09452]. 